\title{
Palliative care and chronic kidney disease in children: a possible dialogue?
}

\begin{abstract}
Chronic kidney disease (CKD) is a problem of great clinical relevance, and is recognized as a complex disease demanding multiple facets in its management. Despite the translation of evidence-based medicine into daily practice has resulted in significant advances in the treatment of CKD, palliative care must be performed simultaneously to the curative treatment, because it is not excluding for the prevention and treatment of the suffering of patients and their families. Therefore, it is necessary to carry out further studies that relate $\mathrm{PC}$ to $\mathrm{CKD}$ for that practice can be evidenced.
\end{abstract}

Keywords: palliative care, chronic kidney disease, children
Volume 2 Issue 3 - 2018

\section{Juliana Ramalho Fernandes, Leopoldo Nelson Fernandes Barbosa}

Department of Psychology, Faculdade Pernambucana de Saúde, Brazil

Correspondence: Juliana Ramalho Fernandes, Department of Psychology, Faculdade Pernambucana de Saúde, Mal. Mascarenhas de Morais Avenue 486I, Imbiribeira, Recife, Pernambuco, Brazil, Tel +5581988309185,

Email julianarfernandes@yahoo.com.br

Received: May 04, 2018 | Published: June II, 2018
Abbreviations: CKD, chronic kidney disease; WHO, world health organization; PC, Palliative care

\section{Introduction}

According to the World Health Organization (WHO) palliative care aims, through multidisciplinary care, improve the quality of life of patients and their families, faced with a disease that threatens the life, through the prevention and relief of suffering, the early identification, assessment and treatment of pain and other physical, social, psychological and spiritual symptoms. ${ }^{1}$ The palliative care (PC) can be applied to any disease condition known to be restrictive of life as, for example, dementia, chronic obstructive pulmonary disease, chronic renal failure, metastatic cancer, cirrhosis, muscular dystrophy, cystic fibrosis, or even, that has a great chance to lead to death as sepsis, multiple organ failure, severe trauma, congenital heart disease. ${ }^{2}$ Chronic kidney disease (CKD) is characterized by a progressive and irreversible loss of renal function, measured from the glomerular filtration rate, specifically when it is below $60 \mathrm{~mL} /$ $\mathrm{min} / 1.73 \mathrm{~m}^{2}$ and for a period equal to or greater than three months with or without renal lesion. ${ }^{3}$

It is a clinical condition that interferes with the daily activities of the person, be they routines of leisure activities, school, work, and the ingestion of liquids and foods, among others that directly affect the quality of life of the people. In children, this condition is even more striking because it directly affects a vital cycle in which the new world is being presented and a good part of the discoveries are monitored by caregivers or intuitive in relation with the other children. The challenges of palliative care with the DRC in this phase are numerous and require constant dialog between family, patient, and multiprofessional team to maintain the well-being and quality of life of children preserved in all its domains: physical, psychological, social, spiritual. ${ }^{4}$

\section{Discussion}

The treatment of CKD should be guided in the early diagnosis of the disease, treatment with interdisciplinary team and implementation of measures able to preserve renal function in the initial stages of the disease. Such measures are necessary to ensure that the patient has chances of survival and quality of life higher and lower rates of morbidity and mortality. ${ }^{5}$ The importance of monitoring in the initial stages of CKD is demonstrated from indices of quality of life and life expectancy higher when compared to the other patients. To this end, it is necessary that there is early referral to specialized service units, preferably with a multidisciplinary team to ensure that the preparation and psychological support to patients and their families can avoid beginning of dialysis treatment, traumatic and that is chosen the best modality of renal replacement therapy. ${ }^{6}$ As the kidney damage progresses, hospitalization becomes necessary due to the renal therapy modality used. This daily coexistence and often prolonged in the hospital environment can cause damage to the psychosocial, physical, cognitive and neurological developments characteristic of the age range of children and adolescents in treatment. ${ }^{4}$

From this, the PC appears as possible before the CKD patients because it is a debilitating disease, inevitably fatal in that there is no possibility of cure. The application of $\mathrm{PC}$ to these patients comes from the time of diagnosis, and may be performed simultaneously to the curative treatment, because it is not excluding for the prevention and treatment of the suffering of patients and their families, practice this neglected in current nephrology. ${ }^{7}$

\section{Conclusion}

The importance of extensive discussion about palliative care as a possibility of care in children diagnosed with CKD is urgent so that quality of life can be offered for patients and families. Therefore, it is necessary to carry out further studies that relate PC to CKD for that practice can be evidenced.

\section{Funding details}

None.

\section{Acknowledgements}

None.

\section{Conflict of interest}

The author declares no conflict of interest. 


\section{References}

1. Cuidados Paliativos. Rio de Janeiro, Brazil: Ministério da Saúde; Instituto Nacional do Câncer; 2012.

2. Weissman DE, Meier DE. Identifying patients in need of a palliative care assessment in the hospital setting - a consensus report from the center to advance palliative care. J Palliat Med. 2011;14(1):17-23.

3. NKF. K/DOQI clinical practice guidelines for chronic kidney disease: evaluation, classification and stratification. Am J Kidney Dis. 2002;39(Suppl 1):S1-246.

4. Soares CMB, Diniz JSS, Lima EM, et al. Chronic kidney disease in children - predialysis interdisciplinary management program. Rev Med Minas Gerais. 2008;18(4 Suppl 1):S90-97.
5. Bastos MG, Kirsztajn GM. Chronic kidney disease: importance of early diagnosis, immediate referral and structured interdisciplinary approach to improve outcomes in patients not yet on dialysis. J Bras Nefrol. 2011;33(1):93-108.

6. Caskey FJ, Wordsworth S, Ben T, et al. Early referral and planned initiation of dialysis: what impact on quality of life? Nephrol Dial Transplant. 2003;18(7):1330-1338.

7. Luz KR, Schmitt PH, Vargas MAO, et al. Palliative care in chronic kidney disease: an integrative review. Enferm Foco. 2013;4(2):75-79. 